\title{
STABILITY OF ENERGY STABLE FLUX RECONSTRUCTION ON GENERALIZED THREE-DIMENSIONAL CURVILINEAR GRIDS FOR LINEAR ADVECTION
}

\author{
Alexander Cicchino $^{1}$, Siva Nadarajah ${ }^{2}$ \\ ${ }^{1}$ Department of Mechanical Engineering, McGill University, Montreal, Canada \\ ${ }^{2}$ Department of Mechanical Engineering, McGill University, Montreal, Canada
}

\begin{abstract}
The flux reconstruction method initially proposed by H.T. Huynh in his seminal paper, has gained popularity in the research community as it recovers promising high-order methods through modally filtered correction fields, such as the Discontinuous Galerkin (DG) method, on unstructured grids over complex geometries. The attraction of the method follows with its stability proofs for the linear advection problem on linear elements, under a class of energy stable flux reconstruction (ESFR) schemes also known as Vincent-Castonguay-Jameson-Huynh (VCJH) schemes. This paper expands the proof to three-dimensional curvilinear elements with nonlinear Jacobians. Additionally, by considering ESFR as a filtered DG scheme, this paper shows a trivial way to solve for the correction functions along faces with nonlinear Jacobians. Also, this paper verifies that the ESFR schemes can in fact be taken as a filtered DG scheme in both strong and weak forms. The main result of this paper is that the energy stability criteria for three-dimensional curvilinear elements results identically to the linear one-dimensional ESFR strong form case for both the ESFR strong and weak forms.
\end{abstract}

Word count: 174 